This is a postprint version of the following published document:

Ayón, X.; Usaola, J. (2015). An optimal scheduling for aggregators in smart grids. En: Institute of Electrical and Electronics Engineers. 2015 12th International Conference on the European Energy Market (EEM) (19-22 May 2015, Lisbon).

DOI: 10.1109/EEM.2015.7216694

(C) 2015. IEEE. Personal use of this material is permitted. Permission from IEEE must be obtained for all other uses, in any current or future media, including reprinting/republishing this material for advertising or promotional purposes, creating new collective works, for resale or redistribution to servers or lists, or reuse of any copyrighted component of this work in other works. 


\section{An Optimal Scheduling for Aggregators in Smart Grids}

\author{
Xiaolin Ayón \\ Universidad Carlos III de Madrid \\ Madrid, Spain \\ xayon@pa.uc3m.es
}

\author{
Julio Usaola \\ Universidad Carlos III de Madrid \\ Madrid, Spain
}

\begin{abstract}
This paper proposes a stochastic optimization method that produces bidding curves to be submitted by an aggregator to the day ahead wholesale markets taking into account the possible imbalance costs as well as the uncertainty of the forecasts (market price, demand and renewable generation for the next day). A formulation is proposed, which yields a linear optimization problem of moderate dimension with short computation times. The result of applying this technique is an increase in revenues of the aggregator from the trading of the resources that it represents. The benefits are assessed, by comparing it with a standard deterministic optimization technique.
\end{abstract}

Index Terms--Distributed energy resource, energy management system, Energy market, smart grid, stochastic programming.

\section{NOTATION}

Indices

$h \quad$ Index of time intervals $1 \ldots N_{h}$.

$d p \quad$ Index of day-ahead prices scenarios, $1 \ldots N_{d p}$.

$r p \quad$ Index of scenarios of renewable production minus fixed demand, $1 \ldots N_{r p}$.

$w \quad$ Index of scenarios of the global random variables, $1 \ldots N_{w}$.

Continuous variables

$P_{n, t}^{d p} \quad$ Total grid net power in period $t$, scenario $d p[\mathrm{~kW}]$.

$\Delta_{t}^{w} \quad$ Total deviation between produced and scheduled energy managed by the aggregator in period $t$, scenario $\mathrm{w}[\mathrm{kW}]$.

$\Delta_{t}^{+w}$ Positive deviation incurred by the aggregator, scenario $w[\mathrm{~kW}]$.

$\Delta_{t}^{-w}$ Negative deviation incurred by the aggregator, scenario $w[\mathrm{~kW}]$.

$P_{d s c h, t}^{d p}$ Total scheduled demand in period $t$, scenario $d p[\mathrm{~kW}]$.

$P_{B, t}^{+d p}$ Power from batteries to grid, period $t$, scenario $d p$ $[\mathrm{kW}]$.

$P_{B, t}^{-d p}$ Power from grid to batteries, period $t$, scenario $d p$ $[\mathrm{kW}]$.

$E_{B, t}^{d p} \quad$ Stored energy in period $t$, scenario $d p[\mathrm{kWh}]$.

\section{Random variables}

$\pi_{t}^{w} \quad$ Day-ahead market price in period $t$, scenario $w$ [€/kWh].

$P_{r n d, t}^{w}$ Renewable production minus noncontrollable demand in period $\mathrm{t}$, scenario $w[\mathrm{~kW}]$.

\section{Constants and data}

$p^{w} \quad$ Probability of occurrence of scenario $w$.

$P_{p v, t}^{w} \quad$ Photovoltaic generation in period $t$, scenario $w[\mathrm{~kW}]$.

$P_{w, t}^{w} \quad$ Wind generation in period $t$, scenario $w[\mathrm{~kW}]$.

$P_{d f i, t}^{w}$ Total fix demand in period $t$, scenario $w[\mathrm{~kW}]$.

$\bar{r}_{t}{ }^{+} \quad$ Ratio between positive imbalance price and day-ahead market price in period $t$.

$\bar{r}_{t}^{-} \quad$ Ratio between negative imbalance price and dayahead market price in period $t$.

$P_{g e n .} \quad$ Installed capacity of the grid $[\mathrm{kW}]$.

$P_{\text {cont. }}$ Contracted power for the grid users. $[\mathrm{kW}]$

$E_{d s c h}$ Daily limit of total scheduled energy [kWh].

$P_{B}^{+\max }$ Maximum power from the batteries $[\mathrm{kW}]$.

$P_{B}^{-\max }$ Maximum power to the batteries [kW].

$E_{B}^{\max }$ Maximum energy in the storage system [kWh].

$E_{B}^{\min }$ Minimum energy in the storage system. [kWh]

$P_{d s c h}^{\max }$ Maximum scheduled demand [kW].

$\eta^{+} \quad$ Efficiency of giving back the stored energy.

$\eta^{-} \quad$ Efficiency of storing energy.

$\Delta E \quad$ Energy losses in storage system [kWh].

$E_{B, 0} \quad$ Initial value of the stored energy [kWh].

\section{INTRODUCTION}

Smart grids will be the future standard at distribution level, after generalization of active demand and distributed generation. The dissemination of those new devices is currently a major challenge for regulators and grid operators, but it also opens a large field of opportunities to use better all the resources available in the grid, in order to achieve a safer, cheaper and more sustainable electric supply.

This work has been supported by European project IDE4L (Ref.: FP7SMARTCITIES-2013-608860) and the Spanish project RESmart (Ref. ENE2013-48690-C2-1-R). 
The aggregator will play a key role in the future regulation of the Smart Grid. This agent might participate in the power market, coordinating consumers, distributed generation resources and storage devices in order to procure energy, sell the energy excess and manage the flexibility of the set of customers represented by it. Its future status is being currently discussed, but its activity could encompass the role of a retailer, a flexibility manager and a balance responsible party or market agent. In the paper we follow this wide definition of the aggregator and study its task of energy procurement for the customers represented by it [1]-[5].

One of the problems faced by these aggregators in this part of its activity is the uncertainty of the consumption and generation of its customers, specially because much of the generation would likely be photovoltaic or nondispatchable, and they will need to use forecasts to submit their bids to the electricity markets. This uncertainty means that the imbalance between the schedule and the actual production/consumption will be high, and this implies imbalance costs. These costs can be reduced using probabilistic optimization methods.

The aggregation of demand and DER allows to gain access to energy markets and hence to maximize the revenue. Additionally, it can be convenient to the system operation [2]. The aggregation concept more extended is the Virtual Power Plant (VPP), which sell, buy or store energy following the price signal.

Uncertainty of DER and market prices are taken into account in different optimization models in order to maximize the aggregator expected profit and reduce its costs. Reference [6] presents a bi-level stochastic model to participate in the power market. Reference [7] proposes a linear stochastic problem for a wind power producer in an electricity market with different trading stages. Reference [8] uses robust optimization (RO) to solve an energy management problem. Reference [9] proposes a stochastic mixed-integer linear programming model to solve the virtual power plant problem. In [10], parallel-distributed optimization OR-X is used to optimize the load schedule of users to minimize the utility company's cost and user payments.

This paper proposes an energy management algorithm to produce optimal bidding curves for the day ahead wholesale markets, taking into account the possible imbalance costs as well as the uncertainty of the forecasts: the market prices, the renewable generation and the demand for the next day. The stochastic approach followed in this work is an extension of [11], leading to a linear formulation of moderate dimension. The objective of applying this technique is to increase the revenues and reduce the cost of the energy purchased by the aggregator. The paper does not deal with the private agreements between the aggregator and its customers, which could be based on long-term contracts.

The optimization problem developed here is set for a customer's portfolio that includes flexible demand, renewables and batteries for a whole day. Then, the best strategy for the management of the flexibility and storage capabilities of this portfolio is chosen.
The method is applied to the benchmark MV grid of CIGRE [12] with distributed generation. The benefits of the proposed method are assessed by comparing it with a standard deterministic optimization technique. The method has been implemented in MATLAB.

\section{SPANISH WHOLESALE ELECTRICITY MARKET AND BALANCING MARKET}

The energy traded in the Spanish wholesale power market, is mostly negotiated in the day-ahead market where purchase and sale bids for day D must be sent to the market operator (OMIE) before the gate closure at 12 a.m. of day D1. The transactions in this pool are settled at the marginal price, available in [13].

In current markets, the TSO is in charge of ensuring a balanced system operation. The energy imbalances between generation and demand are corrected making use of the balancing services, whose costs are covered by those incurring in imbalance. If this is positive, then the energy surplus is paid for their overproduction at the sell imbalance price $\left(\pi_{t}^{+}\right)$lower or equal to the day-ahead market price. On the contrary, if the imbalance is negative, the energy must be bought at the buy imbalance price $\left(\pi_{t}^{-}\right)$higher or equal than the day-ahead market price. The imbalance prices depend on the sign of the net system imbalance. The relation between imbalance and market price may be written in the following way:

$r_{t}^{+}=\frac{\pi_{t}^{+}}{\pi_{t}}, r_{t}^{+} \leq 1 \quad(a) \quad r_{t}^{-}=\frac{\pi_{t}^{-}}{\pi_{t}}, r_{t}^{-} \geq 1$

Although in the future the aggregators might also participate in the balancing market, this possibility is not considered in the present work.

The aggregator objective will be to minimize the cost of trading energy in the day-ahead market taking into account the cost of the imbalance between its scheduled and actual production.

\section{PROBLEM CONSIDERATIONS. ASSUMPTIONS.}

The aggregator considered here manages different generation resources, batteries and demand of different kinds. Further assumptions are: 1) It presents a single bid curve in the daily market for each time interval of the programming horizon (24 hours in the Spanish electricity market) and for the aggregated customers (generation and demand) that the aggregator represents. 2) The aggregator can use storage devices (batteries). And 3) the buy and sell price of the energy are the same. The different kinds of generation and demand are described below.

\section{A. Demand}

Two kinds of demand are considered, namely:

\section{1) Noncontrollable demand.}

This demand might be assimilated to certain manually operated appliances or systems such as lighting, computers, televisions, vacuum cleaners, etc. This type of demand is supplied to the user without restrictions (within the limit of 
the contracted power), but its value is not known beforehand, and the aggregator must forecast it somehow.

For the simulations performed in this paper, trajectories of possible demand prediction with the desired error are generated from a given consumption pattern, taking into account the time dependence of two consecutive time steps. In this way, different possible trajectories of forecasted demand are calculated and used in the optimization process.

\section{2) Scheduled demand (shiftable)}

This demand can be scheduled along a given period (one day, for instance), but the amount of energy to be consumed is fixed. The optimization process described below tends to shift this demand to the hours of the day of lower prices. This kind of demand could be assimilated to electric vehicles, or noncritical devices (e.g., washing machines, dishwashers) [14].

\section{B. Generation}

Only renewable energy is considered in the example, namely solar and wind generation. None of them can be scheduled and hence the aggregator must forecast the production for the considered time interval, that is one day in our formulation.

The solar and wind production forecasts are created in a similar way to the demand forecasting. To a basic pattern of solar and wind production, a random series of the wished variance is superposed. This random series is an $\operatorname{AR}(1)$ in order to preserve the time dependence between two consecutives forecasts.

For the basic pattern of solar production, a site is chosen (latitude and longitude) in order to fit real conditions (hours of sunrise and sunset, amount of solar resource, etc.). The basic trajectory has been obtained according to the method presented in [15]. For the wind production, the basic pattern is the outcome of a real wind farm, normalized to the wished rated power.

\section{Batteries.}

Batteries are modelled in a simple way: maximum and minimum capacity and losses, considered as constant.

\section{Energy and imbalance prices}

The aggregator must perform also a forecast of the energy prices for the day-ahead energy prices. The same procedure as for demand and renewable generation is followed here: to a basic pattern of prices a random series created by means of an $\mathrm{AR}(1)$ series with the desired variance is added. The basic pattern chosen here is the daily market energy price of a given period in the Spanish wholesale market.

Imbalance prices, however, are modelled in a simpler way, since they are extremely volatile. The aggregator assumes a constant ratio between the imbalance and the energy prices. This ratio is different for each hour of the day.

Note that different scenarios of day-ahead prices will result in different scenarios of power to be offered. Then, an offering curve is obtained for each hour. This represents the sensitivity of the ensemble of the aggregator's customers to the price.

\section{E. Scenario tree}

To solve the aggregator problem a two stages stochastic programming is used. The sets of scenarios that characterize the uncertainty can be arranged in a symmetric scenario tree, where the decision framework is given below:

1) In the first stage, $N_{d p}$ price scenarios for the day-ahead market are generated; the first decisions are made according to the market prices and renewable production.

2) For each scenario of the day-ahead market prices, $N_{r p}$ renewable production realizations are generated. This is the second stage where the deviation incurred by the aggregator is known.

The number of total scenarios of the tree are $N_{w}=N_{d p} . N_{r p}$.

\section{IV.MODEL FORMULATION}

The optimization problem that produces the optimal bidding curves to be submitted to the day-ahead market by an aggregator has been formulated as follows:

Max

$\sum_{t=1}^{N_{h}}\left[\sum_{d p=1}^{N_{d p}} p^{d p}\left(\pi_{t}^{d p} P_{n, t}^{d p}\right)+\sum_{w=1}^{N_{w}} p^{w}\left(\pi_{t}^{w} \bar{r}_{t}^{+} \Delta_{t}^{+w}-\right.\right.$

$\left.\left.\pi_{t}^{w} \bar{r}_{t}{ }^{-} \Delta_{t}^{-w}\right)\right]$

subject to

$\sum_{t=1}^{N_{h}} P_{d s c h, t}^{d p}=E_{d s c h}$

$E_{B, t}^{d p}=E_{B, t-1}^{d p}-\frac{1}{\eta^{+}} P_{B, t}^{+d p}+\eta^{-} P_{B, t}^{-d p}-\Delta E, \quad d p=1 \ldots N_{d p}$

$\Delta_{t}^{w}=\left[P_{r n d, t}^{w}-P_{d s c h, t}^{w}+P_{B, t}^{+w}-P_{B, t}^{-w}\right]-P_{n, t}^{w}, w=1 \ldots N_{w}$

$\Delta_{t}^{w}=\Delta_{t}^{+w}-\Delta_{t}^{-w}$

$0 \leq \Delta_{t}^{+^{w}} \leq M P \quad M P=\sum P_{\text {gen }}+\sum P_{\text {cont }}$.

$0 \leq-\Delta_{t}^{-w} \leq M P$

$P_{n, t}^{d p}-P_{n, t}^{d p^{\prime}} \leq 0: \pi_{t}^{d p} \leq \pi_{t}^{d p^{\prime}}$

$P_{r n d, t}^{w}=P_{p v, t}^{w}-P_{d f i, t}^{w}+P_{w, t}^{w}$

$-\sum P_{\text {cont. }} \leq P_{n, t}^{d p} \leq \sum P_{\text {gen }}$.

$0 \leq P_{d s c h, t}^{d p} \leq P_{d s c h}^{\max }$

$0 \leq P_{B, t}^{+d p} \leq P_{B}^{+\max }$

$0 \leq P_{B, t}^{-d p} \leq P_{B}^{-\max }$

$E_{B}^{\min } \leq E_{B, t}^{d p} \leq E_{B}^{\max }$

The objective function (1) is the expected revenue of the aggregator for the participation in the day-ahead market. It includes two terms: The revenue (positive or negative) from the sale or purchase of the energy and a term (positive or negative) due to the imbalance. It must be remarked that only one direction of the net system imbalance is possible. The first term is affected by the daily price probability and the second is affected by the global probability. This probability is calculated assuming the two random variables are independent. This is not strictly right, but the dependence between both variables could be easily considered. 
Equation (2) defines the energy consumed by the scheduled demand, which can be shifted to hours with low electricity prices through the planning horizon. These equations could be reformulated for the case when the charging hours are limited to a given set (e.g., the EV during night). This possibility has not been considered in this paper, but it could be easily included. Equation (3) is the energy balance in the batteries. The charging and discharging cycles have associated efficiencies. The minimum storage losses have been considered constant.

Equation (4) defines the deviation of the aggregator in each period and scenario, i.e., the gap between the energy traded in the day-ahead market and the actual generation. In order to keep the linearity of the problem (5) decomposes the energy imbalance into positive and negative imbalances. This makes possible not to use binary variables since the optimal solution is obtained with $\Delta_{t}^{+w}$ or $\Delta_{t}^{-w}$ equal to zero due the fact that $\bar{r}_{t}^{+} \leq 1$ and $\bar{r}_{t}^{-} \geq 1[11],[16]$.

Constraints (6) and (7) set the limits of the positive and negative deviations. Equation (8) forces the bidding curves to be monotonically increasing accordingly to the rules of the Spanish market. At each hour and scenario the energy prices must be ordered in ascending order. Here, nonanticipativity constraints are not necessary; there will be only one bidding curve for each hour of the day-ahead market. In (9), the random variable $P_{r n d, t}^{w}$ is defined as the renewable production (PV, wind) minus noncontrollable demand. Constraints (10) limit the amount of power that can be purchased or sold in the day-ahead market, according to the grid requirements while (11) - (14) set the bounds of the scheduled demand, storage powers and storage energy, respectively.

\section{CASE Study}

To test the model, we compare it to a conventional deterministic optimization problem whose equations are:

$\operatorname{Max} \sum_{t=1}^{N_{h}} \pi_{t} P_{n, t}$

subject to

Constraints $(2-3),(10-14)$ and

$P_{n, t}=P_{F V, t}-P_{d f i, t}+P_{W, t}+P_{B, t}^{+}-P_{d s c h, t}-P_{B, t}^{-}$

The objective function maximizes the income from the sale of energy or minimizes the cost of purchased energy. Constraints $(2-3)$ and $(10-14)$ are referred to only one scenario and constraints (16) is of power balance.

The data of consumers and generators are taken from the CIGRE benchmark [12]. Certain DG units have not been included (Residential fuel cell, CHP diesel and CHP fuel cell) because they do not add uncertainty to the problem. The total installed power capacity is $1710 \mathrm{~kW}$ with $1500 \mathrm{~kW}$ of wind power and $210 \mathrm{~kW}$ of photovoltaic. The total load is 5291.6 $\mathrm{kW}$, with $3843.6 \mathrm{~kW}$ of noncontrollable demand and 1448 $\mathrm{kW}$ of scheduled demand. The storage consists in two batteries of $600 \mathrm{~kW}$ and $200 \mathrm{~kW}$, and the charge and discharge efficiency is considered as $90 \%$.

The day-ahead market and the imbalance prices have been taken from the Spanish electricity market for the year 2013.
Typical demand profiles have been taken from REE [13]. The total scenarios considered are $10^{6}, 1000$ for day-ahead prices and 1000 for net power (renewable generation minus fixed demand). After a scenario reduction technique, the stochastic problem is formulated with 10 and 20 scenarios for energy prices and net power, respectively.

The prediction scenarios synthesized includes the dependence of the predictions due to the proximity of these customers, since they are located at short distance. Fig. 1(a - b) shows the distribution of the uncertainty of the predictions of prices and of the variable $P_{r n d, t}^{r p}$ for a sample day (01/10/13). Using this uncertainty, the problem gives the bidding curve that would maximize the revenues of the aggregator (or alternatively minimize its purchase costs), not the one that minimizes de imbalance. The results depends on the values of $\bar{r}_{t}{ }^{+}$and $\bar{r}_{t}{ }^{-}$. A good estimate of these parameters is hence important. In the paper, the average hourly value of these parameters in the previous year has been chosen.
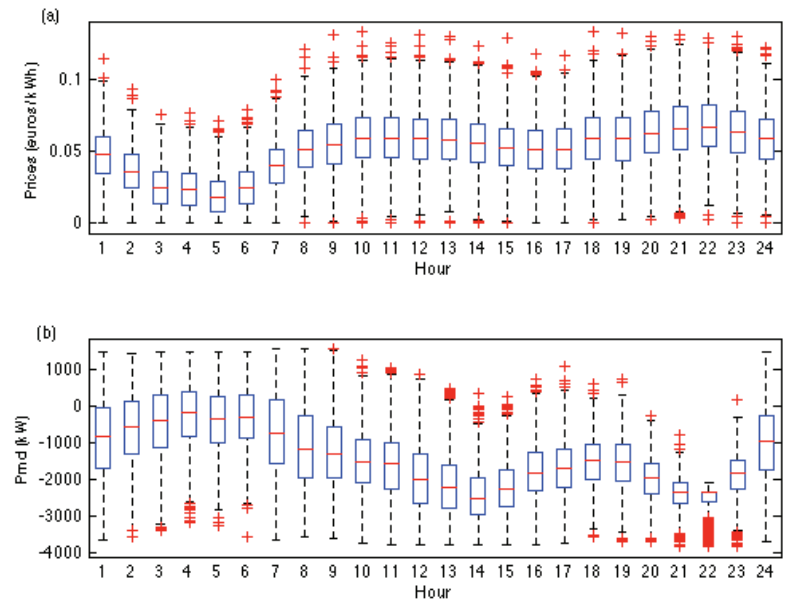

Figure 1. Hourly data forecasts of (a) Day-ahead prices and (b) Renewable production minus fixed demand.

Optimal bidding curves sent to the market for different hours of a sample day are depicted in fig. 2. Each hourly curve consists of ten pairs of values (the number of price scenarios) of energy and marginal price.

The total profit of the aggregator with the proposed stochastic model is better than the deterministic as shown in Table I, for the selected year (2013). This is calculated supposing the day-ahead market has been cleared and a marginal price is agreed. Considering total scenarios as actual forecasts, revenue scenarios for both approaches are generated and the differences per scenario are showed in fig. 3 (a), where the mean of the yearly revenue differences is $€ 3.61 \times 10^{5}$. Fig. 3 (b) illustrates the kernel density estimation of the imbalances registered in both considered approaches, the deterministic imbalances are around the mean, nevertheless, the stochastic exhibits a $\% 75$ of positive imbalances and a $25 \%$ of negatives, i.e. The aggregator will choose to deviate in a positive way in order to have a better profit, it means that imbalance will be negotiated in markets nearest to the deployment of the planning horizon. This demonstrates that 
the stochastic strategy is better than deterministic. The optimization problem was modelled in Matlab 2013a and the computation time for a planning period of 24 hours is around 7 seconds.

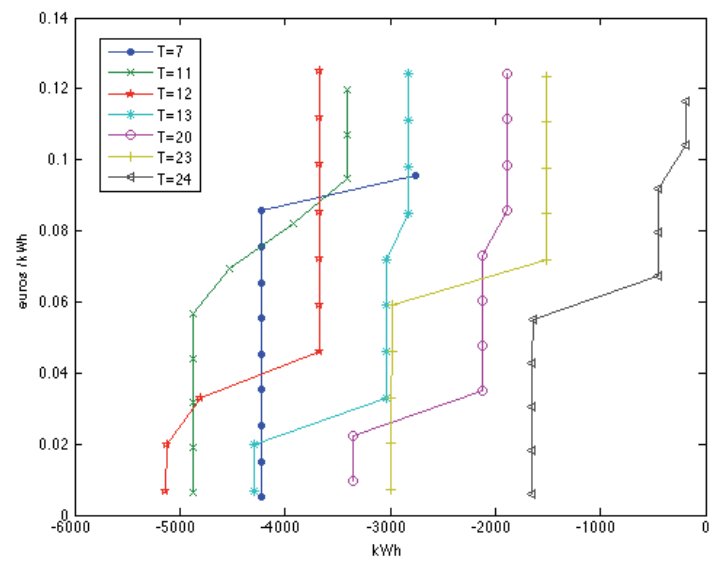

Figure 2. Optimal bidding curves

TABLE I.

EXPECTED PROFIT OF STOCHASTIC AND DETERMINISTIC STRATEGIES

\begin{tabular}{c|c|c}
$\begin{array}{c}\text { Stochastic } \\
(\text { Euro x 10 }\end{array}$ & $\begin{array}{c}\text { Deterministic } \\
(\text { Euro x 10 }\end{array}$ & $\begin{array}{c}\text { Difference } \\
\mathbf{( \% )}\end{array}$ \\
\hline-1.28 & -1.71 & 25
\end{tabular}
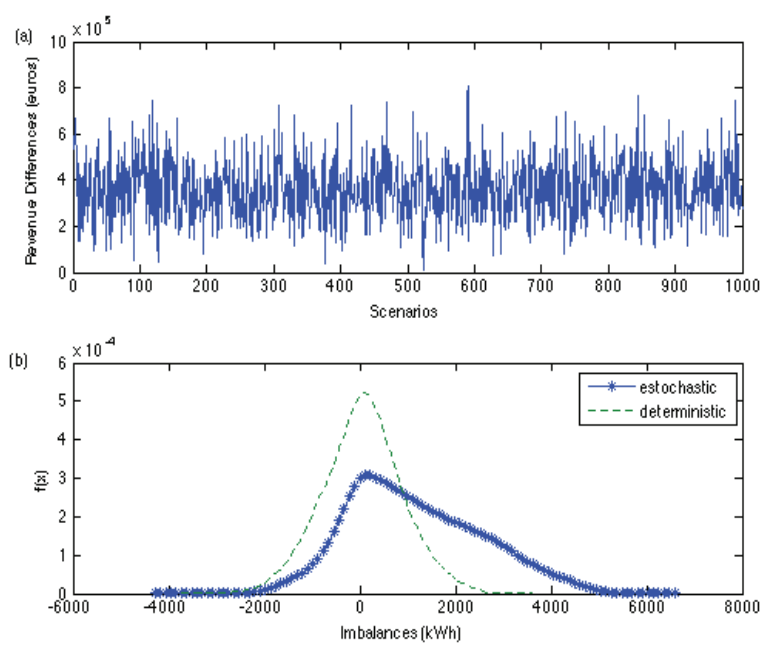

Figure 3. (a) Yearly revenue differences per scenario under the stochastic and deterministic model. (b) The kernel density estimation of the imbalances.

\section{CONCLUSION}

A method for producing optimal bidding curves to the dayahead market for aggregators; taking into account the uncertainty of their available information has been presented. The problem has been modelled as a linear optimization problem of moderate dimension. Different controllable loads, renewable generation and batteries have been considered, and future extensions could be added without special problems.
The revenues for an aggregator participating in the Spanish market for a sample year have been found and compared the results of a conventional deterministic optimization technique, yielding better results.

Future extensions of this work will center on the participation of aggregators in the intraday markets and on the application of this schedule to the individual customers represented by the aggregator.

\section{REFERENCES}

[1] CIRED, "Smart Grids on the Distribution Level - Hype or Vision? " CIRED's point of view, 23.05.2013. Final Report, International Conference on Electricity Distribution.

[2] D. Pudjianto, C. Ramsay and G. Strbac, "Virtual power plant and system integration of distributed energy resources," Renewable Power Generation, IET, vol. 1, no. 1, pp. 10-16, 2007.

[3] Momber, T. Gómez, and L. Söder, "PEV fleet scheduling with electricity market and grid signals", in International Conference on the European Energy Market (EEM), vol. 10th, Stockholm, Sweden: IEEE, May 2013.

[4] M. Carrion, J. Arroyo, and A. Conejo, "A bilevel stochastic programming approach for retailer futures market trading", IEEE Trans. Power Syst., vol. 24, no. 3, pp. 1446 -1456, Aug. 2009.

[5] M. Carrion, A. Philpott, A. Conejo, and J. Arroyo, "A stochastic programming approach to electric energy procurement for large consumers", IEEE Trans. Power Syst., vol. 22, no. 2, pp. $744-754$, May 2007.

[6] Shi, L., Y. Luo, and G. Y. Tu. "Bidding strategy of microgrid with consideration of uncertainty for participating in power market." International Journal of Electrical Power \& Energy Systems, vol. 59, pp. 1-13, 2014.

[7] Morales, J. M., A. J. Conejo, and J. Pérez-Ruiz. "Short-term trading for a wind power producer." IEEE Trans. Power Syst., vol.25, no.1, pp $554-564,2010$

[8] M. Rahimiyan, L. Baringo, A.J. Conejo "Energy Management of a Cluster of Interconnected Price-Responsive Demands". IEEE Trans. Power Syst., vol. 29, no.2, pp. 645 - 655, 2014.

[9] Pandžić, Hrvoje, et al. "Offering model for a virtual power plant based on stochastic programming." Applied Energy 105 (2013): 282-292.

[10] 10P. Yang, P. Chavali, E. Gilboa, A. Nehorai "Parallel Load Schedule Optimization with Renewable Distributed Generators in Smart Grids" IEEE Trans. on Smart Grids, vol.4, no.3, pp. 1431-1441, 2013.

[11] J. Conejo, M. Carrión, J.M. Morales "Decision making under uncertainty in electricity markets", vol. I. New York: Springer, 2010.

[12] K. Rudion, A. Orths, Z. A. Styczynski, and K. Strunz, "Design of benchmark of medium voltage distribution network for investigation of DG integration," presented at the Power Eng. Soc. Gen. Meeting, Montreal, QC, Canada, 2006.

[13] Red Eléctrica de España. [Online]. Available: http://www.esios.ree.es/.

[14] Kim, Tùng T., and H. Vincent Poor. "Scheduling power consumption with price uncertainty." Smart Grid, IEEE Transactions on 2.3 (2011): 519-527.

[15] J. Usaola, V. Ramírez, T. Gafurov, M. Prodanovic "PV modelling for generation adequacy studies". Proceedings of 3rd Solar Integration Workshop. London, October 2013.

[16] M. A. Plazas, A. J. Conejo, and F. J. Prieto. "Multimarket optimal bidding for a power producer," IEEE Trans. Power Syst., vol.20, no.4, pp. 2041-2050, Nov. 2005. 